Krankenhausgesellschaft legt Gutachten vor

\title{
Mangelware Intensivpflegekräfte
}

— Den Kliniken gehen die Intensivpflegekräfte aus. Jedes zweite Krankenhaus (53\%) hat Probleme, entsprechende Stellen zu besetzen. Bei den großen Häusern sind es sogar $68 \%$. Das geht aus einem Gutachten hervor, das die Deutsche Krankenhausgesellschaft (DKG) in Berlin präsentiert hat. Insgesamt sind bundesweit 3.150 Stellen für Intensivpflegekräfte offen. Die vorgegebene Fachkraftquote von mindestens 30\% erreichen drei Viertel der Intensivstationen. Aus Sicht der DKG ist die Patientenversorgung dennoch "objektiv gut“. Eine Pflegevollkraft versorgt laut Gutachten pro Schicht durchschnittlich 2,2 Patienten. Die von der Deutschen Interdisziplinären Verei-

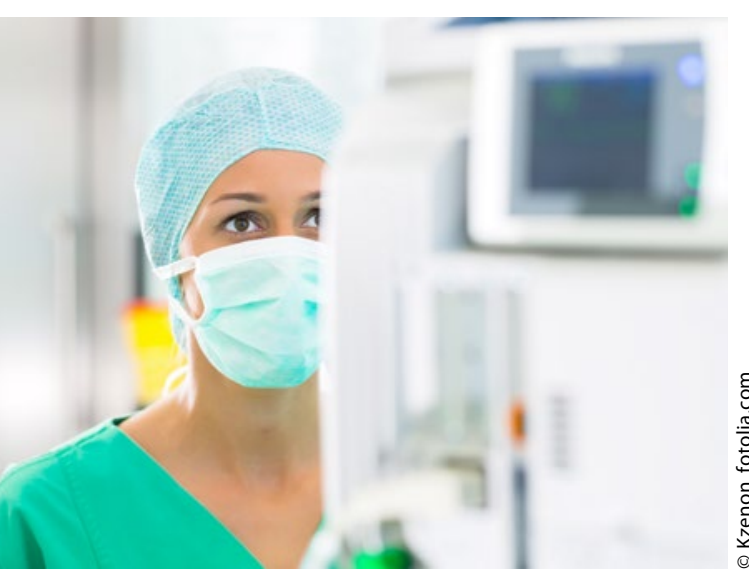

Veranstaltungen des DBfK Nordwest Infos zur Pflegekammer frei Haus

- Der Deutsche Berufsverband für Krankenpflege (DBfK) Nordwest bietet Informationsveranstaltungen rund um Pflegeberufekammern an. Pflegende oder Einrichtungen können sich die Experten kostenlos zu einem Vortrag ins Haus holen. Gut drei Stunden dauern diese Veranstaltungen, der Berufsverband bringt alle Materialien mit. "Hinter dem Angebot steht die Überzeugung, dass nur informierte Pflegende sich eine objektive Meinung zur Pflegeberufekammer bilden können. Diese Erfahrung hat der DBfK in anderen Bundesländern gemacht, in denen die Selbstverwaltung bereits auf den Weg gebracht wurde", sagt nigung für Intensiv- und Notfallmedizin (DIVI) geforderte Quote von zwei Fällen pro Schicht werde damit im Mittel erreicht. Die DKG sieht vor allem Politik und Kostenträger in der Pflicht, dem Fachkräftemangel zu begegnen.

Zweifel an der guten Versorgungssituation hegen sowohl Gewerkschaften als auch Pflegeverbände. „Nichts ist gut in der Intensivpflege", kritisiert Sylvia Bühler, Bundesvorstandsmitglied von Verdi. Aus Berichten von Beschäftigten und vorliegenden Gefährdungsanzeigen werde deutlich, dass ein Verhältnis von 1:3 Pflegekraft-zu-Patienten eher die Regel als eine Ausnahme sei. "Statt den Fachkräftemangel in der Pflege zu beklagen, muss zügig gehandelt werden", erklärt Bühler mit Verweis auf die Arbeitsund Ausbildungsbedingungen. Die DKG sei bei den Verhandlungen zu Personaluntergrenzen gefordert, endlich Nägel mit Köpfen zu machen. Notwendig sind aus Sicht der Gewerkschaft Vorgaben, die Sicherheit für die Patienten und Entlastung für die Beschäftigten bringen.

Die Grundlage für das Gutachten der DKG bildeten die Daten von bundesweit 314 Krankenhäusern. Diese gaben im Herbst 2016 Auskunft zur Personalsituation auf ihren Intensivstationen.

(ne)

www.dkgev.de

Martin Dichter, Vorsitzender des DBfK Nordwest. Es gehe aber auch darum, beruflich Pflegenden Lust auf Politik und Beteiligung zu machen und sie so zu ermutigen, sich in den Pflegekammern zu engagieren. Deshalb sei es wichtig, unkompliziert Veranstaltungen in allen fünf Bundesländern (Bremen, Hamburg, Niedersachsen, Nordrhein-Westfalen, Schleswig-Holstein) anzubieten - unabhängig vom Stand der Entwicklung der Pflegekammer im jeweiligen Bundesland, meint Swantje Seismann, Mitglied des Vorstandes des Berufsverbandes.

www.dbfk.de
Stationäre Behandlung

\subsection{0 neue Stellen in der Pflege}

_ Im Jahr 2016 wurden 19,5 Mio. Patienten stationär im Krankenhaus behandelt. Das waren 277.400 Behandlungsfälle oder 1,4\% mehr als im Jahr zuvor. Wie das Statistische Bundesamt (Destatis) weiter mitteilt, dauerte der Aufenthalt im Krankenhaus wie im Vorjahr durchschnittlich 7,3 Tage. In 1.948 Krankenhäusern Deutschlands standen für die stationäre Behandlung der Patienten insgesamt 498.700 Betten zur Verfügung. Die durchschnittliche Bettenauslastung lag bei $77,8 \%$. Rund 894.500 Vollkräfte - das ist die Anzahl der auf die volle tarifliche Arbeitszeit umgerechneten Beschäftigten versorgten 2016 die Klinikpatienten. Rund 158.000 Vollkräfte gehörten zum ärztlichen Dienst, 736.500 zum nichtärztlichen Dienst, darunter allein 325.200 zum Pflegedienst. Deren Zahl stieg im Vergleich zum Vorjahr um 4.300 .

www.destatis.de

Alles richtig?

Die korrekten Antworten der PflegeKollegs aus der Februar-Ausgabe lauten:

Hygiene leben $1 c, 2 b, 3 c, 4 a, 5 c, 6 a, 7 b, 8 a, 9 b, 10 a$

Ulcus cruris versorgen

$1 a, 2 c, 3 b, 4 a, 5 a, 6 b, 7 c, 8 a, 9 b, 10 c$

Vorschau!

Die PflegeKollegs im Monat Oktober:

Haut pflegen

Altershaut - Handekzem - Lymphödem

Onkologie heute

Sexualität - Ernährung - Medikation 